\title{
Análisis psicosocial de la relación comercial entre vendedores informales y sus consumidores: un estudio en las ciudades de Neiva y Cartagena sobre el comercio informal de café*
}

\section{Psychosocial analysis of the commercial relationship among informal sellers and their consumers: A study carried out in the cities of Neiva and Cartagena about the informal coffee commerce}

\footnotetext{
María Mercedes Botero** Ketty Herrera

\section{Resumen}

La presente investigación tuvo como objetivo fundamental describir las relaciones sociales establecidas entre 60 vendedores informales de café y sus clientes en las ciudades de Neiva y Cartagena (Colombia), teniendo en cuenta la Teoría de Relaciones Sociales de Michael Argyle (1992). Los datos se obtuvieron mediante aproximaciones cualitativas y cuantitativas, con base en técnicas como observaciones, entrevistas y encuestas semiestructuradas. Los resultados indican que las unidades de análisis propuestas en la Teoría de Relaciones Sociales se pueden emplear para así comprender los procesos de interacción entre vendedores informales de café y sus clientes, tomando

Recibido: 7 de noviembre de 2007 Revisado: 4 de diciembre de 2007 Aceptado: 30 de enero de 2008 en cuenta que en cada encuentro se presenta: a) la presencia efectiva de dos actores que se reconocen; b) una asiduidad y permanencia en la interacción que puede ser registrada y cuantificada; c) la realización de una actividad de intercambio que reporta un resultado para cada uno de los actores; y d) un curso en tal interacción.

Palabras clave: comercio informal, café, relaciones sociales, vendedores informales, clientes.

* Proyecto financiado por la Universidad del Norte, Dirección de Investigaciones y Proyectos-Fondo Joven Investigador http://www.uninorte.edu. co/investigacion/secciones.asp?!D=7

** Correspondencia: Ma. Mercedes Botero, Mg., Dirección Postal: Cra. 47 No. 79 - 68, apto. 1A. Barranquilla Colombia. Universidad del Norte, Departamento de Psicología, Correo electrónico: mmbotero@uninorte.edu.co.

*** Correo electrónico: ladygreyjavela@msn.com
} 


\section{Abstract}

The primary objective of this research was to describe the social relationships established in 60 informal coffee sellers and their customers in the cities of Neiva and Cartagena (Colombia), taking as a base the Michael Argyle's Social Relations Theory (1992). Data were obtained through qualitative and quantitative approaches, under observations and semi-structured interviews and surveys techniques. Results indicate that the analysis units proposed in the Social Relations Theory can be used to understand the relationship among informal coffee vendors and their customers, taking into account that in each meeting or interaction among them there is a) the effective presence of two actors that know each other; b) a frequency and a permanence in the interaction that can be registered and quantified; c) an exchange activity that reports a result for each one of the actors; and d) a course in such interaction.

Key words: informal commerce, coffee, social relations, informal vendors, customers.

\section{Introducción}

El comercio informal puede considerarse un fenómeno en el que se realizan actividades que, de una u otra manera, reflejan las condiciones económicas y sociales en las que se encuentra nuestro país, tales como el bajo poder adquisitivo, políticas gubernamentales que buscan la generación de microempresas y cooperativas, desplazamientos forzados masivos y el incremento inusual en las tasas de desempleo y subempleo.

Según el Departamento Nacional de Planeación (2002), el incremento de vendedores informales refleja la delicada situación de la economía colombiana, que se acompaña de "indicadores negativos" y que actualmente está representada por el $35 \%$ de la población total. Al tratarse de una población que está ganando participación en los procesos económicos y sociales de los países subdesarrollados, se convierte en un elemento que merece especial atención en el campo científico, puesto que, de acuerdo con la forma en la que se comprendan las dinámicas sociales que rigen este tipo de procesos en los diversos contextos, se podrá anticipar a manejar adecuadamente algunas situaciones que podrían ser beneficiosas o perjudiciales para la sociedad y ofrecer estrategias de intervención.

La necesidad de indagar sobre las relaciones sociales entre vendedores informales de café y sus consumidores tuvo como objetivo fundamental identificar prácticas comerciales que contribuyan a la incorporación de modelos de acción económica más eficaces $\mathrm{y}$, como consecuencia directa, estimular a que se analice la situación del consumo informal a nivel nacional y sus repercusiones en la economía global.

Mediante la aproximación psicosocial al fenómeno, se busca crear vínculos eficaces que conduzcan a la generación de relaciones sociales leales entre los vendedores y sus consumidores que den cuenta de una relación duradera que permita el beneficio de ambas partes. Por un lado, la prestación de un buen servicio y producto, por parte del vendedor, y por otro, la satisfacción del cliente hacia el producto obtenido, lo cual contribuye a la fidelidad y al cubrimiento de las necesidades de ambas partes.

Las relaciones sociales, al ser un fenómeno que involucra multiplicidad de elementos tanto internos como externos en el ser humano y que se analiza desde la perspectiva de muchas ciencias del conocimiento, permiten iniciar un amplio recorrido en el estudio de la cotidianidad en diversos contextos. Desde la psicología económica vale la pena resaltar los motivos que dan origen a este tipo de relaciones, los actores, temas y situaciones que sobre ellas se construyen.

Además, es importante tener en cuenta que cada espacio en el que se establecen procesos de inter- 
acción, puede tomarse como foco de estudio al tratarse de situaciones en las que confluyen seres humanos que buscan interactuar y compartir su mundo con el de otras personas.

\section{Relaciones sociales, según Michael Argyle}

El tema de las relaciones sociales ha recibido especial atención por parte de diversas áreas del conocimiento, entre las que se hallan la antropología, la etología, la sociología y la psicología (Argyle, 1981; Argyle, Henderson, Bond, Ilzuka \& Contarello, 1986; Argyle, 1992). Ese interés que muestran varias ciencias naturales y sociales en las relaciones sociales se expresa, del mismo modo, en disciplinas que están especialmente interesadas en las relaciones económicas y comerciales; tal es el caso de la administración y el mercadeo (Geiger \& Turley, 2003).

Cada una de estas ciencias hace un aporte significativo y ofrece una manera particular de estudiar el fenómeno. Sin embargo, al tener aproximaciones diversas en naturaleza, técnicas y abordaje, se dificulta la realización de una labor de síntesis que permita integrarlas a todas. Así, por ejemplo, algunos autores emplean el rótulo de relaciones sociales -social relationships-, mientras que otros utilizan el término de relaciones interpersonales -interpersonal relationships- (Blumstein \& Kollock, 1988) y, finalmente, hay quienes hablan de relaciones personales o de relaciones cara a cara.

El panorama que se ofrece para emprender la tarea de sintetizar y valorar los logros alcanzados en el tema de las relaciones sociales llevó a que, en este estudio, se optara por realizar un especial énfasis en la tradición emprendida por el psicólogo social inglés Michael Argyle para alcanzar una visión disciplinar del tema, al tratarse de un autor que presenta en su propuesta un claro compromiso con la psicología social propiamente dicha (Argyle, 1992).
Según Michael Argyle (1992), antes de hablar de relaciones sociales es importante establecer una distinción de lo que se conoce como "contactos sociales", teniendo en cuenta que éstos se caracterizan por ser esporádicos y porque se realizan con personas con quienes es muy poco probable un nuevo encuentro. Por el contrario, las relaciones sociales surgen de un proceso de interacción frecuente entre dos o más personas, en el que es posible observar una serie de fenómenos psicosociales que involucran variables cognoscitivas, afectivas y comportamentales. Cada una de estas relaciones sociales se construye en torno a unos elementos que permiten no sólo caracterizarla y hacerla viable para sus miembros, sino también distinguirla de otro tipo de relaciones sociales.

De acuerdo con este autor, las relaciones sociales empiezan una vez que los "contactos" establecidos entre dos o más personas comienzan a hacerse más frecuentes. Se pueden estudiar considerando las siguientes dimensiones.

\section{a) Actividades y lugares}

Se refiere a todas aquellas cosas que los miembros de la relación hacen durante sus encuentros y los espacios en los que se efectúan. Las actividades que realizan las personas y los espacios en los que las llevan a cabo se convierten en generadores de interacciones sociales. La proximidad física de las personas que diaria o periódicamente confluyen a un lugar común da la posibilidad de reconocer la presencia del otro y el establecimiento de contactos. Si existe frecuencia en los encuentros de tales actores, se pueden generar relaciones sociales.

\section{b) Metas}

Corresponde a los propósitos y a los logros que se plantean y alcanzan los miembros de la relación, es decir, las motivaciones que sostienen las relaciones sociales. La satisfacción encontrada en una relación es el resultado de la ayuda material e instrumental, apoyo social y emocional e intereses comunes. 


\section{c) Reglas}

Operan como normas reguladoras de la interacción al señalar lo que debe y no debe hacerse y, por consiguiente, el seguimiento de esas expectativas de comportamiento es lo que explica el mayor y mejor desenvolvimiento de la relación o su deterioro y disolución. Las reglas son uno de los elementos de las relaciones sociales más importantes (Argyle et al., 1986; Argyle, 1992) y, quizás, en las que se refleja más el contexto social en el que ocurre (Argyle, 1981): "En cualquier cultura hay una serie de reglas y expectativas que rigen la mayor parte de los encuentros sociales [...]" (Argyle, 1981, p. 133).

De acuerdo con Argyle et al. (1986), las reglas en las relaciones sociales que se ocupan del "deber hacer" pueden variar en el número de situaciones que prescriben y las relaciones que cobijan. No obstante, el autor las organiza según dos grandes categorías:

1. Según su propósito: aquellas dirigidas a la interacción o las dirigidas a las metas.

2. Según su carácter: aquellas positivas y las preventivas.

Las conclusiones de Michael Argyle acerca de las reglas que moderan las distintas interacciones sociales son:

1. Todas las relaciones están caracterizadas por una estructura muy ratificada de reglas y sobre ellas existe un consenso entre los sujetos.

2. Ciertas reglas están muy ratificadas en más de la mitad de las relaciones. Son las denominadas "reglas universales".

3. En todas las relaciones orientadas a la tarea, las reglas relacionadas con la tarea se ratifican más altamente que las reglas interpersonales.

4 Las reglas generales se manifiestan en dos tipos de relaciones: las íntimas, que comprenden esposos, familia, amigos y relaciones heterosexuales, y las no íntimas, que incluyen trabajo, relaciones profesionales y de servicios, vecino y relaciones conflictivas.

Algunas de las reglas sociales que operan en las relaciones son cómo nombrar a un miembro de la relación (por su nombre o por su apellido, por ejemplo); celebración de cumpleaños, pago de cuentas, visitas sociales o invitaciones familiares, uso de palabras afectivas, contacto físico, etc.; cuáles son los temas que se deben tratar y cuáles son aquellos que, por el contrario, se deben evadir (Argyle et al., 1986).

En cuanto a los temas tratados en una determinada relación, las relaciones sociales se pueden clasificar así:

1. Relaciones con alta confidencia y baja variabilidad en temas.

2. Aquellas con alta confidencia y alta variabilidad.

3. Las de baja confidencia y baja variabilidad.

4. Las relaciones con baja confidencia y alta variabilidad.

\section{d) Habilidades}

Son todas aquellas competencias de interacción que despliega un miembro de la relación cuando se encuentra con otro(s). Las habilidades no se restringen a aquellos despliegues de conducta que se realizan cuando se siguen las reglas, también incluyen aquellos comportamientos que permiten actuar exitosamente en el momento de inicio, mantenimiento y disolución de la relación.

\section{e) Conceptos y creencias}

Son todas aquellas nociones que poseen los miembros de una relación acerca de la misma. Tales nociones son el producto de diferentes experiencias de aprendizaje, como por ejemplo las derivadas de la experiencia directa o las adquiridas por aprendizaje social (donde los medios masivos cumplen un papel definitivo). 


\section{f) Poder y roles}

Énfasis en la capacidad de influencia que tienen los miembros de la misma para sustentar la noción de poder y diferenciación en los roles que pueden llegar a caracterizar una determinada relación social.

\section{Relaciones sociales entre un vendedor y su cliente}

En la sociedad contemporánea es evidente el alto nivel de interdependencia que alcanzan sus miembros a la hora de satisfacer sus metas; los tiempos de los individuos o los primeros grupos autosuficientes pertenecen al pasado de la humanidad.

Una buena razón del alto nivel de interdependencia que actualmente alcanzan los humanos, se origina en el complejo tramado que constituye la sociedad contemporánea; esto, a su vez, conduce a que todos sus miembros desempeñen tareas cada vez más especializadas.

El comercio es una de esas actividades especializadas de la sociedad contemporánea (Vinchur, Schippmann, Switzer y Roth, 1998). Quienes desempeñan esta tarea procuran servir de enlace entre aquellos encargados de transformar recursos (productores de bienes y servicios), y aquellos que los requieren para su diario transcurrir (consumidores de bienes y servicios).

Del buen o mal dominio y, por consiguiente, del desempeño de las tareas implicadas en las actividades de comercialización no sólo depende el éxito económico y social de quienes realizan dicha labor, sino que también dependen de ello los trabajadores, empleadores e inversionistas de las industrias que proveen los bienes y servicios que se van a transar (Richins, 1983 y Vinchur et al., 1998); y, finalmente, de la manera en que nos comportamos con los otros en el momento de la interacción; de este aspecto se puede aprender mediante un modelo o una elección consciente en lo que respecta al comportamiento.
Al determinar las consecuencias probables de estos comportamientos y al computar las probabilidades de que los costos o los beneficios asociados con los diferentes comportamientos tengan lugar en la realidad, se selecciona el mejor comportamiento para lograr una mejor interacción social. La centralidad de dicho ejercicio de comercialización en la economía contemporánea ha conducido a una inversión intensa y extensa por parte de las empresas en los factores que forman parte del mercadeo de bienes y servicios, incluyendo en esos costos la capacitación de los recursos humanos implicados en dichos procesos (Vinchur et al., 1998).

Esa fuerte inversión en el mercadeo de bienes y servicios y, naturalmente, en la capacitación de recursos humanos ha conducido a una alta diversificación y especialización de la oferta en los distintos sectores y categorías de productos.

Los académicos que se ocupan del tema han desarrollado un número escaso de formulaciones (Richins, 1983), las cuales, sin embargo, presentan una gran variedad de aproximaciones al tema de la comercialización y de los agentes implicados en ella, aunque en un número pequeño de formulaciones sistemáticas. En términos generales, esas distintas propuestas conceptuales se pueden organizar en tres grandes enfoques: los centrados en el vendedor (ver, por ejemplo, Richins, 1983 y Vinchur et al., 1998), los que se ocupan de la situación y, finalmente, los que se especializan en las relaciones vendedor-cliente.

Aunque existen varias formas de organizar las actividades comerciales, aquí se está abordando un caso muy específico de intermediación que se observa en el sector informal de la economía: aquella que desempeñan los vendedores informales ambulantes. Éstos son, como otros tipos de comerciantes, personas cuya actividad económica está relacionada con la intermediación, pero a diferencia de los sectores formales, éstos no cuentan con un local o espacio para la realización de sus actividades, ni están sujetos a todos los procesos de formalización social (Martínez, 1999); $y$, en el caso de otros tipos de comercio informal, se desplazan por distintos puntos de una zona que ellos pretenden cubrir con sus servicios. 
Los vendedores informales no estacionarios pueden contar con medios distintos para el transporte de sus mercancías en sus recorridos, desde aquellas personas que utilizan carruajes de tracción humana, quienes cuentan con carrozas de tracción animal -por ejemplo, zorras- hasta aquellas que recurren a vehículos motorizados, como motos y automóviles (Martínez, 1999).

Acorde con la naturaleza de los productos por ellos ofrecidos, algunos comerciantes informales ambulantes pueden establecer recorridos que duran unas horas (por ejemplo, proveedores de café, cigarrillos y dulces, abastecedores de alimentos), o aquellos que cubren varios meses (por ejemplo, vendedores de utensilios para el hogar) o, incluso, aquellos que recurren a medios de transporte motorizados.

Los vendedores informales no estacionarios son personas que generalmente se ocupan de la comercialización de una relativamente bien definida categoría de productos y, del mismo modo, en cada uno de sus renglones suelen conformar un surtido que garantiza una oferta variada pero delimitada de bienes (Martínez, 1999), incluyendo en esa oferta, claro está, una serie de productos complementarios y sustitutos.

Durante su diario quehacer, los vendedores informales ambulantes desarrollan una actividad que implica emprender varios contactos sociales con el propósito de ofrecer sus servicios a potenciales compradores que encuentra en su ruta y cuando logra identificar la presencia de un(os) interesado(s) en el (los) bien(es) o servicio(s) que ofrece, establecer una interacción que culmine con la utilización de sus servicios y, en el mejor de los escenarios posibles, preparar el terreno para conformar una relación duradera con el comprador (construir una relación de lealtad).

La presencia de compradores esporádicos y compradores permanentes o leales en el recorrido de los vendedores informales no estacionarios señala la existencia de dos grandes clases de interacción: una primera, caracterizada por una secuencia de actos sin perspectiva en el tiempo y carente de dinámicas específicas de los actores involucrados, y una segunda, que se distingue por configurarse en la dimensión temporal e incorpora aspectos propios de los partícipes de la relación.

Blumstein y Kollock (1988) ofrecen varios elementos que pueden distinguir los dos tipos de interacciones: por un lado, las de los compradores esporádicos y, por el otro, la de los clientes leales; de acuerdo con ellos:

1. Las relaciones con los clientes leales son interacciones enmarcadas por una serie de eventos ocurridos en el pasado (desde el primer contacto) y configuradas por una serie de expectativas sobre episodios en el futuro.

2. Las relaciones que establece el vendedor con cada uno de sus clientes leales llegan a poseer propiedades específicas y, por lo tanto, distintivas de las otras interacciones.

3. Las relaciones con los clientes leales no sólo se distinguen por esas propiedades "únicas", que emergen de la interacción, sino que también implican la expresión de las individualidades de los actores participantes y, por consiguiente, se otorga una mayor particularidad. Así, mientras que los contactos con compradores esporádicos implican interacciones en las que priman roles y posiciones sociales que puede desempeñar y ocupar cualquier persona -cualquier vendedor y cualquier comprador-, las interacciones con clientes leales responden a ejecuciones sociales específicas y para nada regidas por agentes y libretos estereotipados.

En el mercadeo contemporáneo, una meta fundamental de todo proceso es el establecimiento y mantenimiento de relaciones sociales duraderas con quienes, a partir de ellas, se constituyen en sus clientes leales (Young y Denize, 1995). El establecimiento de relaciones duraderas entre un vendedor y sus compradores genera varias ventajas para el vendedor; la principal se refiere a que una vez establecida una lealtad, los compradores preferirán a su proveedor habitual sobre otras ofertas que pueden, incluso, ofrecer mayores ventajas (Young y Denize, 1995). 


\section{Metodología}

\section{Objetivo general}

Describir las características de las relaciones sociales de los vendedores ambulantes informales y sus consumidores en las ciudades de Neiva y Cartagena (Colombia).

\section{Objetivos específicos}

1. Identificar las características de las relaciones sociales desde el modelo propuesto por Michael Argyle (1992): Actividades y lugares, poder y roles, reglas, habilidades, conceptos y creencias, y metas, de los vendedores ambulantes informales y sus consumidores en la ciudad de Neiva (Colombia).

2. Identificar las características de las relaciones sociales desde el modelo propuesto por Michael Argyle (1992): Actividades y lugares, poder y roles, reglas, habilidades, conceptos y creencias, y metas, de los vendedores ambulantes informales y sus consumidores en la ciudad de Cartagena (Colombia).

3. Comparar las características de las relaciones sociales de los vendedores ambulantes informales y sus consumidores en las ciudades de Neiva y Cartagena (Colombia).

\section{Tipo de estudio}

La presente investigación corresponde a un estudio de tipo cuantitativo, específicamente de corte descriptivo comparativo. El propósito fundamental fue la explicación de un fenómeno en dos contextos diferentes, que para el caso corresponde a las relaciones sociales establecidas entre vendedores informales y clientes, con base en la teoría propuesta por Michael Argyle. Tal fenómeno se analizó en cada contexto en particular: Cartagena (ubicada en una amplia bahía de islas y lagunas en la costa caribe colombiana) y Neiva (ubicada entre las cordilleras Central y Oriental, en una planicie sobre la margen oriental del río
Magdalena), y posteriormente se compararon los resultados entre sí.

\section{Población y muestra}

La población con la que se trabajó corresponde a los vendedores informales de café y sus clientes en las ciudades de Cartagena y Neiva (Colombia). De esa población se seleccionó una muestra de 60 vendedores y 240 clientes, 110 identificados como frecuentes o leales y 130 como esporádicos.

\section{Instrumentos}

Teniendo en cuenta las unidades de análisis que caracterizan las relaciones sociales, según Michael Argyle, se construyeron los instrumentos de medición para la presente investigación. El checklist contiene los registros de las observaciones de campo, mediante los cuales se obtuvo información relacionada con variables temporales, asociadas a las reglas, habilidades y metas. Los cuestionarios para el vendedor y para el cliente tuvieron como objetivo fundamental indagar acerca de variables asociadas a las actividades y lugares, creencias y metas de interacción. El mapa cognitivo permitió obtener información sobre las rutas de acción comercial seguidas por los vendedores de café, identificación de sitios clave, clientes y creencias sobre el ambiente de trabajo y roles sociales.

\section{Procedimiento}

La recolección de la información en la ciudad de Cartagena se hizo en universidades, parques históricos y sitios turísticos de la ciudad. Antes de aplicar los instrumentos se procedió a realizar una observación en algunos sectores de la ciudad, con el porpósito de ubicar lugares estratégicos para abordar a los vendedores. Luego de las observaciones de campo, se procedió a establecer los contactos con la muestra. Para esta fase hubo que ir a algunas cafeterías de la ciudad, con el fin de hablar con las personas y pedir su consentimiento para acompañarlas en su recorrido. Una vez realizados los contactos, se daba cita al 
inicio de la jornada (mañana o tarde) y el investigador caminaba con el vendedor, observando las conductas y los hechos que se explicitaron en los objetivos de la investigación y algunos otros aspectos relevantes del entorno.

Durante el recorrido, el investigador iba entrevistando al vendedor y se indagaba sobre aspectos referentes al recorrido seguido durante el desarrollo de la actividad comercial y la relación con clientes fieles y esporádicos. Adicionalmente, solicitaba información sobre los clientes fieles y esporádicos y de acuerdo con ello se aplicaba la entrevista al cliente. Adicional a ello se efectuaban las observaciones que se consignarían en la lista de chequeo.

El primer paso seguido en la ciudad de Neiva fue la realización de observaciones durante varios días antes de la recolección de la información. En ellas se determinó el flujo de personas, los lugares en los que se ubican los vendedores de café, las rutas seguidas por algunos de ellos y el tipo de clientes que acuden diariamente a comprar el producto.

Inicialmente, se identificaron seis personas, que sirvieron como apoyo para obtener el resto de la muestra. En esta ciudad no hay cafeterías, por lo que el contacto se estableció de manera personal con cada vendedor participante.

El investigador siempre llegaba donde el vendedor y se sentaba a su lado. Si no había clientes, procedía con la aplicación de la encuesta al vendedor, pero si llegaba alguno, se interrumpía la entrevista, con el propósito de recolectar los datos de la lista de chequeo. Una vez que se tenían tales datos, el vendedor identificaba a uno de los clientes más frecuentes, a quien se hacía la entrevista.

Ahora bien, en la recolección para los clientes no estacionarios se seguía otro proceso. Como primera medida, el investigador se encontraba con el vendedor en el parque, con el objeto de iniciar juntos el recorrido. A medida que iban siguiendo la ruta (que siempre era en lugares cercanos al parque) se iba realizando el mapa cognitivo y aplicando la entrevista al vendedor. Si durante el proceso algún cliente solicitaba los productos, se suspendía el proceso para registrar datos de la lista de chequeo. Cuando el vendedor identificaba un cliente frecuente, el investigador procedía a la aplicación de la entrevista a éste, finalizando así el proceso de recolección.

\section{Resultados}

Entre las actividades y lugares del proceso de interacción entre los vendedores y clientes en las ciudades estudiadas, se hallaron similitudes en aspectos relacionados con la solicitud/ofrecimiento de los productos, la cual se hace de manera familiar, caracterizándose en algunos casos por gestos como sonrisas, miradas o palabras. Este proceso se singulariza, principalmente, porque es el vendedor el que ofrece a los clientes los productos y no son ellos los que buscan a su proveedor. Normalmente los vendedores conversan con los clientes y hacen énfasis en temas relacionados en especial con el trabajo.

Los lugares en los que se establecen los procesos de interacción entre los vendedores informales de café y clientes en la ciudad de Cartagena se realizan en el parque Bolívar, zona centro y zona de playas, donde se realizan actividades de manera estacionaria y semiestacionaria, principalmente. En la ciudad de Neiva predomina el comercio informal estacionario en el parque Santander, sobre otro grupo que establece rutas en sectores cercanos al parque.

Entre las metas que orientan la relación entre vendedores informales y clientes en las ciudades estudiadas, los resultados indican que existen similitudes en lo relacionado con las estrategias de fidelización, puesto que en ambas muestras de clientes se identificó el buen trato como un elemento de vital relevancia en la recompra de productos al mismo vendedor. Sin embargo, las metas de atracción a nuevos clientes, por parte de los vendedores en la ciudad de Neiva, se orientan hacia la buena atención en un $30 \%$, mientras que en la ciudad de Cartagena para el $46,6 \%$ de los 
clientes es importante que el vendedor les ofrezca el producto.

En cuanto a las metas relacionadas con la compra de productos, los resultados indican que en la ciudad de Neiva un $30 \%$ de la muestra de clientes considera que compra siempre al mismo vendedor por la calidad de los productos, mientras que en la ciudad de Cartagena el $30 \%$ de la muestra consideró que lo hace por la cercanía a su lugar de trabajo o vivienda.

Las reglas que orientan la interacción entre los vendedores informales de café en las ciudades estudiadas no se basan en saludos verbales. Las personas manifiestan contacto visual o saludo gestual antes de iniciar el proceso de interacción con los clientes, y en la mayoría de los casos es el vendedor el que emprende las pautas de interacción. En el saludo no se emplean seudónimos; no obstante, durante la interacción se establece un clima de respeto.

Una vez finalizado el proceso de comercialización, los clientes no se alejan del vendedor. En caso contrario, permanecen al lado del vendedor -aunque no todas las veces- y siguen hablando con ellos. En ocasiones los vendedores permanecen cerca de los clientes mientras ellos conversan con otras personas. Se encontraron diferencias en la familiaridad del saludo por parte de los clientes, identificando en la ciudad de Neiva un 57,8\% de favorabilidad y en Cartagena un $65,6 \%$ que no saluda a los vendedores con familiaridad.

En lo relacionado con las habilidades, los resultados indican que el $100 \%$ de los clientes entrevistados en la ciudad de Neiva consideran que los vendedores poseen habilidades para la preparación de lo que comercializan. "Tienen los termos y puestos limpios y organizados", "Buen punto de azúcar y café”, “Están pendientes de que el café sea como el cliente lo quiere", "Productos frescos", manifiestan. En la ciudad de Cartagena, el $86,6 \%$ de la muestra de clientes considera que el vendedor posee habilidades para la preparación de los productos. La mayoría de los clientes en ambas ciudades no solicitan instrucciones al vendedor sobre la preparación de los productos ni tampoco los vendedores solicitan indicaciones sobre la manera de preparar los productos.

Los conceptos y creencias de más del $50 \%$ de los vendedores, en cuanto al saludo, indican que el cliente es el que debe saludar al vendedor. En el caso de los clientes, los resultados indicaron que en la ciudad de Neiva un 53,3\% de la muestra espera que sea el vendedor el que salude, mientras que en la ciudad de Cartagena el $73,3 \%$ es quien espera que el vendedor salude.

Las expectativas de saludo en ambas ciudades se definen por la cortesía, que implica el uso de expresiones de saludo como "buenos días", "buenas tardes", según sea el caso. Las maneras en las que los clientes esperan que el vendedor salude en la muestra de vendedores, en la ciudad de Cartagena, se orientan en un $63 \%$ hacia fines afectivos, mientras que en la ciudad de Neiva son importantes sobre todo la educación y los modales del vendedor al momento de interactuar.

Las creencias relacionadas con la atracción de nuevos clientes por parte de los vendedores en la ciudad de Cartagena se orientan, en un $76,6 \%$, al ofrecimiento del producto, que se realiza mediante silbidos, llamados, gritos o canciones, mientras que en la ciudad de Neiva las respuestas estuvieron centradas en una buena atención o no existe alguna estrategia (30\%).

En cuanto al poder y roles, se encontró que un 57,8\% de los clientes en la ciudad de Neiva es amable con el vendedor, principalmente al saludarlo y al dirigirse a él, mientras que en la ciudad de Cartagena un $60 \%$ de la muestra no es amable con el vendedor.

Los roles comerciales desempeñados por los vendedores en ambas ciudades se orientan especialmente a la calidad de productos y acceso a vendedores, lo que contribuye al incremento en las ventas.

\section{Conclusiones}

Desde el punto de vista de Michael Argyle (1992), las relaciones sociales se constituyen cuando los 
contactos sociales dejan de ser hechos sociales aislados y empiezan a convertirse en interacciones que ocurren con mayor periodicidad y con los mismos participantes. Al considerar como un tipo de relación social la establecida entre vendedores informales de café y sus clientes e intentar analizarla desde las categorías propuestas por la Teoría de Relaciones Sociales, se pudo observar que efectivamente las unidades de análisis propuestas por el autor resultan útiles para elaborar una aproximación psicosocial a las transacciones realizadas por estos actores sociales.

Las interacciones establecidas entre los vendedores informales de café con sus clientes se pueden llegar a calificar como relaciones sociales si se considera que los resultados señalaron:

a) La presencia efectiva de dos actores que se reconocen.

b) Una asiduidad y permanencia en la interacción que se puede registrar y cuantificar.

c) Una actividad de intercambio que reporta un resultado para cada uno de los actores.

d) La descripción de un curso en la interacción.

En el contexto de la relación vendedor-comprador, un buen número de aspectos de la interacción de géneros se encuentra aún inexplorado. Se puede especular si los compradores buscan vendedores que compartan su mismo sistema de valores, lo que podría incluir los rasgos de personalidad estereotípicos del género. No es claro si los compradores buscan cualidades en un consejero financiero, como la empatía, que no poseen ellos mismos.

\section{Otros hallazgos relacionados con la literatura revisada}

El establecimiento de relaciones duraderas entre un vendedor y sus compradores genera varias ventajas para el vendedor; la principal es que una vez que se establece lealtad, los compradores prefieren a su proveedor habitual sobre otras ofertas que, incluso, pueden ofrecer mayores ventajas (Young y Denize, 1995). La lealtad no sólo se basa en las bondades del servicio ofrecido. Con frecuencia, un factor determinante de la lealtad del comprador hacia un vendedor en particular es el relacionado con los costos que implica emprender una nueva interacción con otro proveedor (Young y Denize, 1995).

\section{Referencias}

Argyle, M. (1981). Psicología del comportamiento interpersonal. Madrid: Alianza Universidad.

Argyle, M. (1992). Relaciones sociales. En M. Hewstone, W. Stroebe, J. Codol \& M. Stephenson (eds.). Introducción a la Psicología: una perspectiva europea. Barcelona: Ariel Psicología.

Argyle, M., Henderson, M., Bond, M., Ilzuka, Y. \& Contarello, A. (1986). Cross-cultural variations in relationship rules. International Journal of Psychology, 21, 287-315.

Blumstein, P. \& Kollock, P. (1988). Personal relationships. Annual Review of Sociology, 14, 467-490.

Departamento Nacional de Planeación (noviembre de 2002). ¿Quiénes conforman el sector informal en Colombia? Cartillas de Economía, 2, 2-4.

Martínez, R. (1999). Estrategias para sobrevivir. Inmigrantes en la venta ambulante. Intervención Psicosocial, 8 (2), 251-263.

Richins, R. (junio de 1983). An analysis of consumer interaction styles in the marketplace. Journal of Consumer Research, 10, 73-82.

Vinchur, A. J., Schippmann, J. Switzer III, F. S. \& Roth, P. L. (1998). A meta-analytic review of predictor of performance for salespeople. Journal of Applied Psychology, 83 (4), 586597. 
Young, L. \& Denize, S. (1995). A concept of commitment: alternative views of relational continuity in business service relationships. Journal of Business \& Industrial Marketing, 10 (5), 22-37. 